\title{
Examples of Freudenthal-Kantor triple systems
}

\section{Noriaki Kamiya*}

Department of Mathematics, University of Aizu, Aizuwakamatsu, 965-8580, Japan

\author{
Abstract \\ Symmetry group of Lie algebras and superalgebras constructed from $(\epsilon, \delta)$ Freudenthal-Kantor triple systems has \\ been studied. Also, the definition and examples of hermitian triple systems is introduced in this note.
}

\section{AMS classification: 17C50; 17A40; 17B60}

Keywords: Triple systems; Lie algebras; Symmety group

\section{Introduction}

It seems that the concept of a triple system (or called a ternary algebra) in nonassociative algebras started from the metasymplectic geometry due to Freudenthal. After a generalization of the concept has been studied by Tits, Koecher, Kantor, Yamaguti, Allison and authors $([1,2]$ for many earlier references on the subject). Also it is well known the object of investigation of Jordan and Lie algebras with application to symmetric spaces or domains [3] and to physics [4,5].

Nonassociative algebras are rich in of mathematics, not only for pure algebra differential geometry, but also for representation theory and algebraic geometry. Specialy, the Lie algebras and Jordan algebras plays an important role in many mathematical and physical objects. As a construction of Lie algebras as well as Jordan algebras, we are interested in characterizing the Lie algebras from view point of triple systems [69]). These imply that we are considering to structure of the subspase $\mathrm{L}_{1}$ of the five graded Lie (super)algebra $L(\epsilon, \delta)=L_{-2} \oplus L_{-1} \oplus L_{0} \oplus L_{1} \oplus L_{2}$ satisfying $\left[\mathrm{L}_{\mathrm{i}}, \mathrm{L}_{\mathrm{j}}\right] \subseteq \mathrm{L}_{\mathrm{i}+\mathrm{j}}$, associated with an $(\epsilon, \delta)$ Freudenthal-Kantor triple system which contains a class of Jordan triple systems related 3 graded Lie algebra $\mathrm{L}_{-1} \oplus \mathrm{L}_{0} \oplus \mathrm{L}_{1}$. For these consideretions without untilizing properties of root systems or Cartan matrices, we would like to refer to the articles of the present author and earlier references quoted therein $[1,2,10,11]$.

In particular, for an characterizing of Lie algebras, an applying to geometry and physics, we will introduce couple topics about a symmetry of Lie algebras (section 1) and a definition of hermitian triple systems (section 2) in this note, which is a survey and an announcement of new results.

More precisely speaking, first, the symmetry group of Lie algebras and superalgebras constructed from $(\epsilon, \delta)$ Freudenthal-Kantor triple systems has been studied. Especially, for a special $(\epsilon, \epsilon)$ FreudenthalKantor triple, it is $S L$ (2) group. Secondly, we will give a definition of hermitian * generalized Jordan triple systems and the examples of their tripotents defined by elements $C$ of triple systems satisfying $C C C=C$.

\section{Symmetry of Lie algebras associated with triple sytems}

A triple system $V$ is a vector space over a field $F$ of characteristic $\neq 2$ or 3 with a trilinear map $V \otimes V \otimes V \rightarrow V$. We denote the trilinear product ( or ternary product) by juxtaposition $(x y z) \epsilon$ $V($ or $<x y z>,[x y z], e t c)$.

A well studied triple system is the $(\epsilon, \delta)$ Freudenthal-Kantor triple systems ( abbreviated hereafter as to $(\epsilon, \delta)$ FKTS) with $\epsilon$ and $\delta$ being either +1 or $-1[1,2,9,12]$.
Since $(\epsilon, \delta)$ Freudenthal-Kantor triple systems offer a simple method of constructing Lie algebras (for the case of $(\delta=+1)$ ) and Lie superalgebras (for the case of $\delta=-1$ ) with 5 -graded structure, it may be of some interest to study its symmetry group in this note. In order to facilitate the discussion, let us briefly sketch its definition.

We introduce two linear mappings $L$ and $K: V \oplus V \rightarrow$ End $V$ by

$$
L(x, y)=x y z, K(x, y) z=-\delta y z x(1.1)
$$

for $\delta=+1$ or -1 If they satisfy

$$
\begin{aligned}
& {[L(u, v), L(x, y)]=L(L(u, v) x, y)+\epsilon L(x, L(v, u) y),(1.2)} \\
& K(K(u, v) x, y)=L(y, x) K(u, v)-\epsilon K(u, v) L(x, y)(1.3)
\end{aligned}
$$

for any $u, v, x, y \in V$ and $\epsilon=+1$ we call the triple system to be $(\epsilon, \delta)$ FKTS.

One consequence of Eqs.(1.2) and (1.3) is the validity of the following important identity (see [Y-O.] Eqs.(2.9) and (2.10))

$$
\begin{aligned}
& K(u, v) K(x, y)=\epsilon \delta L(K(u, v) y, x-\epsilon L(K(u, v) x, y)(1.4) \\
& =L(v, K(x, y) u)-\delta L(u, K(x, y) v)(1.5)
\end{aligned}
$$

We note that $(-1,1)$ FKTS is said to be a generalized Jordan triple system of second order [12] in section 2, becaue that is a generalization of concept of Jordan triple systems.

We can then construct a Lie algebra for $\delta=+1$ and a Lie superalgebra for $\delta=-1$ as follows:

Let $W$ be a space of $2 \times 1$ matrices over $V$

$W=\left(\begin{array}{l}V \\ V\end{array}\right)$

and define a tri-linear product:

$W \oplus W \oplus W \rightarrow W$ by

$$
\begin{aligned}
& {\left[\left(\begin{array}{l}
x_{1} \\
y_{1}
\end{array}\right),\left(\begin{array}{l}
x_{2} \\
y_{2}
\end{array}\right),\left(\begin{array}{l}
x_{3} \\
y_{3}
\end{array}\right)\right]=} \\
& \left(\begin{array}{cc}
L\left(x_{1}, y_{2}\right)-\delta L\left(x_{2}, y_{1}\right) & \delta K\left(x_{1}, x_{2}\right) \\
-\varepsilon K\left(y_{1}, y_{2}\right) & \varepsilon L\left(y_{2}, x_{1}\right)-\varepsilon \delta L\left(y_{1}, x_{2}\right)
\end{array}\right)\left(\begin{array}{l}
x_{3} \\
y_{3}
\end{array}\right) .(1.6)
\end{aligned}
$$

*Corresponding author: Norikai kamiya, Department of Mathematics, University of Aizu, Aizuwakamatsu, 965-8580, Japan, Tel: +0242-37-2500; E-mail: kamiya@u-aizu.ac.jp

Received January 26, 2014; Accepted June 02, 2014; Published June 10, 2014

Citation: Kamiya N (2014) Examples of Freudenthal-Kantor triple systems. J Generalized Lie Theory Appl 8: 210. doi: 10.4172/1736-4337.1000210

Copyright: ( 2014 Kamiya N. This is an open-access article distributed under the terms of the Creative Commons Attribution License, which permits unrestricted use, distribution, and reproduction in any medium, provided the original author and source are credited. 
Then, it defines a Lie triple system for $\delta=+1$ and an anti-Lie triple system for $\delta=-1$ We then note

$$
\hat{L}_{\overline{0}}=\operatorname{span}\left\{\left(\begin{array}{cc}
L(x, y) & \delta K(z, w) \\
-\varepsilon K(u, v) & \varepsilon L(y, x)
\end{array}\right) \mid x, y, z, w, u, v \in V\right\}(1.7)
$$

is a Lie subalgebra of $\mathrm{Mat}_{2}(\operatorname{End}(V))^{-}$where $B$ for an associative algebra $B$ implies a Lie algebra with bracket; $[x, y]=x y$ - $y x$ We note also then

$$
\hat{D}=\left(\begin{array}{cc}
L(x, y), & \delta K(z, w) \\
-\varepsilon K(u, v), & \varepsilon L(y, x)
\end{array}\right) \in L(W, W)(1.8)
$$

is a derivation of the triple system. Setting

$$
L_{\overline{1}}=\operatorname{span}\left\{X=\left(\begin{array}{l}
x \\
y
\end{array}\right) \mid x, y \in V\right\}(=W),(1.9
$$

$$
L=L_{\overline{0}} \oplus L_{\overline{1}}(1.10)
$$

gives a Lie algebra for $\delta=+1$ and a Lie superalgebra for $\delta=-1$ where

$$
L_{\overline{0}}=\{D \mid D \text { is a derivation of } L\},(1.11)
$$

i.e., $D$ satisfies

$$
\begin{aligned}
& =D\left[X_{1}, X_{2}, X_{3}\right] \\
& {\left[D X_{1}, X_{2}, X_{3}\right]+\left[X_{1}, D X_{2}, X_{3}\right]+\left[X_{1}, X_{2}, D X_{3}\right](1.12 a)}
\end{aligned}
$$

and hence induces also

$$
[D,[X, Y]]=[D X, Y]+[X, D Y],(1.2 b)
$$

if we define the bracket by

$$
\left[D_{1} \oplus X_{1}, D_{1} \oplus X_{2}\right]=\left(\left[D_{1}, D_{2}\right]+L\left(X_{1}, X_{2}\right)\right) \oplus\left(D_{1} X_{2}-D_{2} X_{1}\right) .
$$

where

$$
\left[\mathrm{D}_{1}, \mathrm{D}_{2}\right]=\mathrm{D}_{1}, \mathrm{D}_{2-} \mathrm{D}_{2}, \mathrm{D}_{1}
$$

and

$$
\begin{aligned}
& L\left(X_{1}, X_{2}\right)=\left[X_{1}, X_{2}\right]=\left[\left(\begin{array}{l}
x_{1} \\
y_{1}
\end{array}\right),\left(\begin{array}{l}
x_{2} \\
y_{2}
\end{array}\right)\right]= \\
& \left(\begin{array}{cc}
L\left(x_{1}, y_{2}\right)-\delta L\left(x_{2}, y_{1}\right) & \delta K\left(x_{1}, x_{2}\right) \\
-\varepsilon K\left(y_{1}, y_{2}\right) & \varepsilon L\left(y_{2}, x_{1}\right)-\varepsilon \delta L\left(y_{1}, x_{2}\right)
\end{array}\right) .
\end{aligned}
$$

Note that the endomorphism $L(X, Y)$ is then an inner derivation of the triple system.

Since $L_{\overline{0}} \supset \hat{L}_{\overline{0}}$, we will mainly discuss a subsystem $\hat{L}$ of $L$, given by rather than the larger $L$. Then, $\hat{L}$ is 5 -graded

$$
\hat{L}=L_{-2} \oplus L_{-1} \oplus L_{0} \oplus L_{1} \oplus L_{2}(1.16)
$$

where

$$
\begin{aligned}
& L_{-2}=\operatorname{span}\left\{\left(\begin{array}{cc}
0 & 0 \\
-\varepsilon K(x, y) & 0
\end{array}\right) \mid x, y \in V\right\}(1.17 a) \\
& L_{-1}=\operatorname{span}\left\{\left(\begin{array}{l}
0 \\
x
\end{array}\right) \mid x \in V\right\}(1.17 b) \\
& L_{0}=\operatorname{span}\left\{\left(\begin{array}{cc}
L(x, y) & 0 \\
0 & \varepsilon L(y, x)
\end{array}\right) \mid x, y \in V\right\}(1.17 c) \\
& L_{1}=\operatorname{span}\left\{\left(\begin{array}{l}
x \\
0
\end{array}\right) \mid x \in V\right\}(1.17 d)
\end{aligned}
$$

$$
L_{2}=\operatorname{span}\left\{\left(\begin{array}{cc}
0 & \delta K(x, y) \\
0 & 0
\end{array}\right) \mid x, y \in V\right\} \cdot(1.17 e)
$$

Here, we utilized the following Proposition for some of its proof.

Theorem 1: ([K-O.], [K-M-O.]) Let $(V,(x y z)))$ be a $(\epsilon, \delta)$ -Freudenthal-Kantor triple system with an endomorphism $P$ such that $P^{2}=-\epsilon \delta I d$ and $P(x y z)=(P x P y P z)$ Then, $(V,[x y z)]$ is a Lie triple system (for $\delta=1)$ and anti-Lie triple system (for $\delta=-1)$ with respect to the product

$[x y z]=(x P y z)-\delta(y P x z)+\delta(x P z y)-(y P z x)$.

In passing, we note that the standard $\hat{L}=\Sigma_{i=-2}^{2} \oplus L_{i}$ is a result of Theorem 1 immediately with

$$
P=\left(\begin{array}{cc}
0, & \delta \\
-\varepsilon, & 0
\end{array}\right) \text { and } x \rightarrow X \text { etc. }
$$

Next, we introduce $\theta, \sigma(\lambda) \in \operatorname{End}(\hat{L})$ for any $\lambda \in F(\lambda \neq 0)$, being non-zero constant by

$$
\begin{aligned}
& \theta\left(\begin{array}{l}
x \\
y
\end{array}\right)=\left(\begin{array}{c}
-\varepsilon y \\
\delta x
\end{array}\right)(1.18 a) \\
& \sigma(\lambda)\left(\begin{array}{l}
x \\
y
\end{array}\right)=\left(\begin{array}{l}
\lambda x \\
\frac{1}{\lambda} y
\end{array}\right)(1.18 b)
\end{aligned}
$$

in $W=L_{\overline{\mathrm{T}}}=L_{-1} \oplus L_{1}$. We may easily verify that they are automorphism of $[W, W, W]$, i.e, we have for example

$$
\theta([X, Y, Z])=[\theta X, \theta Y, \theta Z]
$$

for $X, Y, Z \in W$ We then extend their actions to the whole of $\hat{L}$ in a natural way to show that they will define automorphism of $\hat{L}$. They moreover satisfy

(i) $\sigma$

$\sigma(1)=I d, \theta^{4}=\operatorname{Id}(1.19 a)$

where Id is the identity mapping

(ii)

$\theta^{2}=-\varepsilon \delta I d$ for $L_{\overline{1}}$ but $\theta^{2}=I d$ for $L_{\overline{0}}(1.19 b)$

(iii)

$\sigma(\mu) \sigma(v)=\sigma(\mu v)$ for $\mu, v \in F, \mu v \neq 0$

(iv)

$\sigma(\lambda) \theta \sigma(\lambda)=\theta$ for any $\lambda \in F, \lambda \neq 0 .(1.19 d)$

We call the group generated by $\sigma(\lambda)$ and $\theta$ satisfying these conditions simply as $\mathrm{D}(\epsilon, \delta)$ due to a lack of better terminology. If the field $F$ contains $\omega \in F$ satisfying $\omega^{3}=1$ but $\omega \neq 1$, then a finite sub-group of $\mathrm{D}(\epsilon, \delta)$ generated by $\theta$ and $\sigma(\omega)$ defines $\mathrm{DiC}_{3}$ group for $\epsilon=\delta$ but $S_{3}$ for $\epsilon=-\delta$, as they have noted already [13].

Conversly any 5 -graded Lie algebra (or Lie superalgebra) with such automorphism $\theta$ and $\sigma(\lambda)$ satisfying Eqs.(1.19) lead essentially to a $(\epsilon \delta)$ FKTS in $L$, with a triple product defined by $\{x, y, z\}=[[x, \theta y] z$, for $x, y, z \in L_{1}[6]$.

We note that the corresponding local symmetry of $\mathrm{D}(\epsilon, \delta)$ yields a derivation of $L$, given by

$$
h=\left(\begin{array}{cc}
1 & 0 \\
0 & -1
\end{array}\right)(1.20)
$$


which satisfies

$$
h[X, Y, Z]=[h X, Y, Z]+[X, h Y, Z]+[X, Y, h Z](1.21 a)
$$

as well as $[h,[X, Y]]=[h X, Y]+[X, h Y](1.21 b)$ for $X, Y, Z \in W$.

We can find a larger automorphism group of $\hat{L}$, if we impose some additional conditions. First suppose that $K(x, y)$ is now expresed as

$$
K(x, y)=\epsilon \delta L(y, x)-\epsilon L(x, y)(1.22)
$$

for any $x, y \in V$. We call then the triple system to be a special $(\epsilon, \delta)$ FKTS [13]. Moreover for the case of special $(\epsilon, \epsilon)$ FKTS (i.e. $\epsilon=\delta$ ), the automorphism group of $L$ turns out to be a larger $S L(2, F)(=S p(2, F))$ group which contains $D(\epsilon, \epsilon)$ as its subgroup. In this case, the triple system $[W, W, W]$ becomes invariant under

$$
\left(\begin{array}{l}
n \\
y
\end{array}\right) \rightarrow U\left(\begin{array}{l}
n \\
y
\end{array}\right)(1.23 a)
$$

for any $2 \times 2 S L(2, F)$ matrix $U$,i.e.

$$
U=\left(\begin{array}{ll}
\alpha & \beta \\
\mu & v
\end{array}\right), \operatorname{Det} U=\alpha v-\beta \mu=1 .(1.23 b)
$$

Also, the associated Lie algebras or superalgebras are $\mathrm{BC}_{1}$-graded algebra of type $\mathrm{C}_{1}$

Finally, we consider a ternary system $(V, x y, x y z)(V, x y, x y z)$ where $x y$ and $x y z$ are binary and ternary products, respectively, in the vector space $V$. Suppose that they satisfy

(1) the triple system $(V, x y z)$ is a $(-1,1)$ FKTS.

(2) The binary algebra $(V, x y)$ is unital and involution $(\overline{x y}=\overline{y x})$ with the involutive map

(3) The triple product $x y z$ is expressed in terms of the bi-linear products by

$$
x y z=(z \bar{y}) x-(z \bar{x}) y+(x \bar{y}) z .(1.24)
$$

We may call the ternary system $(V, x y, x y z)$ to be Allison-ternary algebra or simply $A$-ternary algebra, since $A=(V, x y)$ is then the structurable algebra [14-16]. This case is of great interest, first because structurable algebras exhbit a triality relation [16,17], and second because we can construct another type of Lie algebras independently of the standard construction of $(-1,1)$ FKTS, which is $S_{4}$-invariant and of $B C_{1}$ graded Lie algebra of type $B_{1}$. The relationship between the Lie algebra constructed in the new way and that given as in Eq.(1.17) is by no means transparent. Note that the group $D(-1,1)$ contains $S_{3}$ but not $\mathrm{S}_{4}$ symmetry. We may show that if the field $F$ contains the square root $\sqrt{-1} \in F$, of-1, then Eqs.(1.17) can be prolonged to yield the Lie algebra for the structurable algebra.

The Lie algebras constructed as in Eqs.(1.13) and (1.14) is also a $B C_{1}$-graded Lie algebra of type $B_{1}$ without assuming $\sqrt{-1} \in F$, ([6]). Also, if $F$ is an algebraically closed field of characteristic zero, then any simple Lie algebra is known to be $\mathrm{S}_{4}$-invariant and can be constructed by some structurable algebra, so that any such Lie algebra is also a $B C_{1}$ graded Lie algebra of type $B_{1}$, (as well as of type $C_{1}$ ). Of course, the underlying $s l(2)$ symmetry is different for both $B_{1}$ and $C_{1}$ cases. Roughly speaking, it seems that these concept are a version of Lie algebras theory corresponding with a Galois group of algebraic numbers theory.

The contents of this section is a cowork with Prof. Okubo, and the details will be discussed in other papers.

\section{Hermitian triple systems}

For a geometrical object based on triple systems in this section, first we note that the symmetric bounded domains are a one to one, correspondence to hermite Jordan triple systems, such that a certain trace form is positive definite hermitian. Hence as a generalization of these triple systems, we are interesting to investigate for structure theory of hermitian generalized Jordan triple systems, in particular, the case of hermitian generalized Jordan triple systems of second order (i.e. hermitian (-1,1) -Freudenthal-Kantor triple system ).

We shall concerned with algebras and triple systems which are infinite over a complex number field, unless otherwise specified in this section.

Definition: A triple system $V$ is said to be $a^{*}$-generalized Jordan triple system of second order if relations (0)--(iv) satisfy;

0) $V$ is a Banach space,

i) $[L(a, b), L(c, d)]=L(\langle a b c\rangle, d)-L(c,<b a d\rangle)$,

ii) $K(<a b c>, d)+K(c,<a b d>)+K(a, K(c, d) b)=0$,

where $L<(a, b) c=\langle a b c\rangle$ and $K(a, b) c=\langle a c b\rangle-\langle b c a\rangle$,

iii) $<x y z>$ is $C$-linear operator on $x, z$ and $C$-anti-linear operator on $y$,

iv) $<a b c>$ continuous with respect to a norm \|\| that is, there exists

$K>0$ such that

$\|<x x x>\| \leq K\|x\|^{3}$ for all $x \in V$.

Furthermore ${ }^{*}$-generalized Jordan triple system of second order is said to be hermitian if it satisfies the following condition,

v) all operator $L(x, y)$ is a positive hermitian operator with a hermitian metrix

$$
(x, y)=\operatorname{trace} L(x, y),(2.1)
$$

that is, $(L(x, y) z, w)=\left(z, L^{*}(x, y) w\right)$, and $(x, y)=\overline{(y, x)}$. In particular, if a triple system $V$ satisfies the condition (o),(i),(iii),(iv) and (v), then it is said to be a hermitain ${ }^{*}$-generalized Jordan triple system.

Furthermore, as a generalization of the generalized Jordan triple system of second order, it is said to be a . $(\epsilon, \delta)$-Freudenthal-Kantor triple system if the following relations satisfy;

$$
\begin{aligned}
& \text { i) ' }[L(a, b), L(c, d)]=L(<a b c>, d) \epsilon L(c,<b a d>) \\
& \text { ii) ' } K(<a b c>, d)+K(c,<a b d>)+\delta K(a, K(c, d) b)=0,
\end{aligned}
$$$$
\text { where } K(a, b) c=\langle a b c\rangle-\delta\langle b c a\rangle, \epsilon= \pm 1, \delta= \pm 1 \text {. }
$$

We note that these identities $(i)^{\prime}$ and $(i i)^{\prime}$ are equivalent to the identities (1.2) and (1.3) in section $1[2,7]$.

Example 1: Let $V$ be a $J^{*}$-triple (for the definition, to Loos [3]. Then $V$ is a hermitian ${ }^{*}$-generalized Jordan triple system of second order, because they satisfy the condition (i) and a special case of (ii) , that is,

$$
\mathrm{K}(\mathrm{x}, \mathrm{y}) \mathrm{z}=\langle\mathrm{xyz}>-<\mathrm{yzx}>\equiv 0
$$

(identicallly zero).

Example 2: Let $T_{n, n}^{*}$ be the space of diagonal matrix of $\mathrm{n} \times \mathrm{n}$ with element of the complex number. Then $T^{*}$ is a ${ }^{\star}$-generalized Jordan triple system of second order, with respect to the product and the norm

\| $\|$

$<x y z>=x \bar{y}^{T} z+z \bar{y}^{T} x-\bar{y} x^{T} z$ 


$$
\begin{aligned}
& \text { for all } x, y, z \in T_{n, n}^{*} \\
& \|x\|=\max \left(\left|\lambda_{i}\right|,\left|\mu_{i}\right|\right)
\end{aligned}
$$

where $x=\Sigma\left(\lambda_{i} e_{i}+\mu_{i} \sqrt{-1} e_{i}\right), \lambda_{i}, \mu_{i} \in R$ (real number) and $\mathrm{e}_{\mathrm{i}}$ are matrix unit element of $T_{n, n}^{*}$ and $x^{T}$ is the transpose of $x$.

Next let $V$ be a ${ }^{*}$-generalized Jordan triple system. Then we may define the notation of a tripotent and a bitripotent as follows.

Definition: It is said to be a tripotent of $V$ if

$\langle c c c\rangle=c, c \in V(2.2)$.

Definition: It is said to be a strongly bitripotent of $V$ if a pair $\left(c_{1}, c_{2}\right)$ of

$\left\langle c_{1} c_{1} c_{2}\right\rangle=-1 / 2 c_{2},\left\langle c_{1} c_{1} c_{2}\right\rangle=-1 / 2 c_{1}$,

and other porducts are zero.

Definition: It is said to be a bitripotent of $V$ if a pair $\left(c_{1}, c_{2}\right)$ of the tripotens satisfy the relations

$$
\begin{aligned}
& \left\langle c_{1} c_{1} c_{2}\right\rangle=\alpha c_{2},\left\langle c_{2} c_{2} c_{1}\right\rangle=\alpha c_{1}, \\
& \left\langle c_{1} c_{2} c_{1}\right\rangle=\beta c_{2},\left\langle c_{2} c_{1} c_{2}>=\beta c_{1},\right. \\
& \left\langle c_{2} c_{1} c_{1}\right\rangle=\gamma c_{2},\left\langle c_{1} c_{2} c_{2}>=\gamma c_{1},\right.
\end{aligned}
$$

and other products are zero, where

$\alpha^{2}+\beta^{2}+\gamma^{2} \neq 0, \alpha, \beta, \gamma \in R$ (real number).

For *-generalized Jordan triple system $V$, we can define a norm || || as follows;

$$
\|x\|=\max \left|\lambda_{i}\right|, i f x=\Sigma \lambda_{i} e_{i} \in V,
$$

where $e_{i}$ are tripotents or bitripotents. We note that

$$
\|x\| \geq 0 \text { and }\|x+y\| \leq\|x\|+\|y\| \text {. }
$$

Example 3: (a generalization of Example 2.2) Let $D^{*}{ }_{n, k}$ be the set of all $\mathrm{n} \times \mathrm{k}$ matrices with operation

$<x y z>:=x \bar{y}^{T} z+z \bar{y}^{T} x-\bar{y} x^{T} z,(2.3)$

where $x^{T}$ and $\bar{x}$ mean transpose and conjugation of $x$ respectiverly.

Then $D_{n k}^{*}$ is a hermitian *-generalized Jordan triple system of second order. In fact, the conditions (i),(ii) and (iii) are evident, by straightfoward calculations.

For (v) considering, we have $(x, y):=\operatorname{tr}(x, y)$ linear on $x$ and anti linear on $y$ by the condition (iii). Thus we may get $\operatorname{tr} L(x, y)=\operatorname{tr} \overline{L(y, x)}$ as follows.

From the relations;

$$
\begin{aligned}
& (L(x, y)+L(y, x)) z=x \bar{y}^{T} z+z \bar{y}^{T} x-\bar{y} x^{T} z+y \bar{x}^{T} z+z \bar{x}^{T} y-\bar{x} y^{T} z \\
& =\left(x \bar{y}^{T}+y \bar{x}^{T}\right) z-\left(\bar{x} y^{T}+\bar{y} x^{T}\right) z+z\left(\bar{y}^{T} x+\bar{x}^{T} y\right), \\
& (L(x, y)-L(y, x)) z=x \bar{y}^{T} z+z \bar{y}^{T} x-\bar{y} x^{T} z-y \bar{x}^{T} z-z \bar{x}^{T} y+\bar{x} y^{T} z \\
& =\left(x \bar{y}^{T}-y \bar{x}^{T}\right) z+\left(\bar{x} y^{T}-\bar{y} x^{T}\right) z+z\left(\bar{y}^{T} x-\bar{x}^{T} y\right), \\
& =\left(x \bar{y}^{T}-y \bar{x}^{T}\right) z+\left(\bar{x} y^{T}-\bar{y} x^{T}\right) z+z\left(\bar{y}^{T} x-\bar{x}^{T} y\right),
\end{aligned}
$$

it follows that $L(x, y)+L(y, x)$, and $L(y, x)-L(y, x)$ have are a real trace form and an imagenary trace form respectively. Thus we have $(x, y)=\overline{(y, x)}$.

To prove positive definite, we consider

$L(x, x) z=x \bar{x}^{T} z+z \bar{x}^{T} x-\bar{x} x^{T} z=\left(x \bar{x}^{T}-\bar{x} x^{T}\right) z+z \bar{x}^{T} x$.

Since $\operatorname{tr} x \bar{x}^{T}=\operatorname{tr} \bar{x} x^{T}$, we get trace $L(x, x)=k \operatorname{trace} \bar{x}^{T} x$, this means that the trace from $(x, y)$ is positive definite. It is enough to show the condition (iv). By means of result of the property of matrix, we can write

$$
x=\Sigma \mu_{i} e_{i},\|x\|=\max \left|\mu_{i}\right|,
$$

$e_{i}$ are tripotents or bitripotens if $e_{i}$ is the unit element of matrix..

Moreprecisely speaking, we have

$\|x\|=\max \left(\left|\lambda_{i j}\right|,\left|\mu_{i j}\right|\right)$ for $x=\Sigma\left(\lambda_{i j} E_{i j}+\mu_{i j} \sqrt{-1} E_{i j}\right)$,

where the notations are denoted by $\lambda i j, \mu i j \in R$ (real number field) and also $\mathrm{E}_{\mathrm{ij}}$ means that $(i, j)$ element is 1 and othere element is zero. $\mathrm{E}_{\mathrm{ij}}$ and $\sqrt{-1} E_{i j}$ are tipotents, i.e., $\left\langle\mathrm{E}_{\mathrm{ij}} \mathrm{E}_{\mathrm{ij}} \mathrm{E}_{\mathrm{ij}}\right\rangle=\mathrm{E}_{\mathrm{ij}}$, and $\left\langle\sqrt{-1} E_{i j} \sqrt{-1} E_{i j} \sqrt{-1} E_{i j}>=\sqrt{-1} E_{i j}\right.$. Furthermore, we note $\left(E_{i j}, \sqrt{-1} E_{i j}\right)$ are bitripotents.

Then we have

$\|<x x x>\|=\left\|2 x \bar{x}^{T} x-\bar{x} x^{T} x\right\| \leq 2\left\|x \bar{x}^{T} x\right\|+\left\|\bar{x}^{T} x\right\| \leq 3\|x\|^{3}$.

These show that $D_{n, k}^{*}$ is a hermilian *-generalized Jordan triple system of second order, that is, a hermitian $(-1,1)$-FKTS.

Examples 4: Let $S^{*}{ }_{2 n, \mathrm{k}}$ be the set of all $2 n \times k$ matrices with operation

$<X Y Z>=X(\bar{Y})^{T} Z+Z(\bar{Y})^{T} X-\phi(\bar{Y}) \phi(X)^{T} Z(2.4)$

where $X=\left(\begin{array}{l}x_{1} \\ x_{2}\end{array}\right), x_{i}$ is an $n \times k$ - matrix, and $(\quad)=(\quad)$.

Then $S^{*}{ }_{2 n, k}$ is a hermitian *-generalized Jordan triple system of second order.

In fact, the elements $\frac{1}{\sqrt{2}}\left(\begin{array}{c}E_{i j}^{(1)} \\ E_{i j}^{(2)}\end{array}\right) \in S_{2 n, k}^{*}$ are tripotents.

For any element $\mathrm{X}$ of $\mathrm{S}_{2 \mathrm{n}, \mathrm{k}}$,we may represent as follows;

$X=\Sigma\left(\lambda_{i j}^{(1)} E_{i j}^{(1)}+\mu_{i j}^{(1)} \sqrt{-1} E_{i j}^{(1)}+\Sigma\left(\lambda_{l m}^{(2)} E_{l m}^{(2)}+\mu_{l m}^{(2)} \sqrt{-1} E_{l m}^{(2)}\right)\right.$

and the norm is defined by

$$
\|X\|=\max \left(\left|\lambda_{i j}^{(1)}\right|,\left|\lambda_{l m}^{(2)}\right|,\left|\mu_{i j}^{(1)}\right|,\left|\mu_{l m}^{(2)}\right|\right)
$$

where $\mathrm{E}_{\mathrm{ij}}{ }^{(1)}$ is the matrix unit of $\left(\begin{array}{c}S_{n, k} \\ 0\end{array}\right)$ and $E_{l m}^{(2)} \mathrm{E}_{\mathrm{lm}}{ }^{(2)}$ is the matrix unit of $\left(\begin{array}{c}0 \\ S_{n, k}\end{array}\right)$

By straightforward calculations as well as Example 2.3, we can show that $S^{\star}{ }_{2 n, \mathrm{k}}$ is a hermitian ${ }^{\star}$-generalized Jordan triple system of second order. Example 4: Let $\mathrm{A}_{\mathrm{kn}}^{*} \oplus \mathrm{A}_{\mathrm{nl}}^{*} \mathrm{~A}_{\mathrm{kn}}^{*}$ be the set fo all pairs $X=\left(\begin{array}{l}x_{1} \\ x_{2}\end{array}\right)$,
where $x_{1}$ is a $k \times n$ matrix and $x_{2}$ is a

$n \times l$ matrix with operation given by foumula

$$
<X Y Z>=\left(\begin{array}{c}
x_{1} \bar{y}_{1}^{T} z_{1}+z_{1} \bar{y}_{1}^{T} x_{1}-z_{1} x_{2} \bar{y}_{2}^{T} \\
x_{2} \bar{y}_{2}^{T} z_{2}+z_{2} \bar{y}_{2}^{T} x_{2}-\bar{y}_{1}^{T} x_{1} z_{2}
\end{array}\right)
$$

Then $\mathrm{A}_{\mathrm{kn}}^{*} \oplus \mathrm{A}_{\mathrm{nl}}^{*}$ is a hermitian ${ }^{*}$-generalized Jordan triple system of second order.

In fact, for $X=\left(\begin{array}{l}u_{1} \\ u_{2}\end{array}\right)$. we have

$(X, X)=\operatorname{trace} L(X, X)=n$ trace $u_{1} \bar{u}_{1}^{T}+l$ trace $u_{2} \bar{u}_{2}^{T}$,

hence it follows that the trace form $($,$) is positive definite.$

For any element of $\mathrm{A}_{\mathrm{kn}}^{*} \oplus \mathrm{A}_{\mathrm{nl}}^{*}$, we may represent as follows;

$X=\Sigma\left(\lambda_{i j}^{(1)} E_{i j}^{(1)}+\mu_{i j}^{(1)} \sqrt{-1} E_{i j}^{(1)}\right)+\Sigma\left(\lambda_{s t}^{(2)} E_{s t}^{(2)}+\mu_{s t}^{(2)} \sqrt{-1} E_{s t}^{(2)}\right)$ 
and $\left(\mathrm{E}_{\mathrm{ij}}^{(1)}, \mathrm{E}_{\mathrm{jt}}^{(2)}\right) \epsilon \mathrm{A}_{\mathrm{kn}}^{*} \oplus \mathrm{A}_{\mathrm{nl}}^{*}$ is a tripotent.

Furthermore, the norm is defined by

$$
\|X\|=\sqrt{2} \max \left(\left|\lambda_{i j}^{(1)}\right|,\left|\lambda_{s t}^{(2)}\right|,\left|\mu_{i j}^{(1)}\right|,\left|\mu_{s t}^{(2)}\right|\right) \text {. }
$$

Example 6: Let $\mathrm{C}^{*}{ }_{\mathrm{nn}} \oplus \mathrm{A}_{\mathrm{nl}}^{*}$ be the set of all pairs $X=\left(\begin{array}{l}x_{1} \\ x_{2}\end{array}\right)$, where $x_{1}$ is a skew-symmetric $n \times n$ matrix and $x_{2}$ is a column,i.e.,is a $n \times 1$ matrix, with operation given by formula,

$$
<X Y Z>:=\left(\begin{array}{c}
x_{1} \bar{y}_{1}^{T} z_{1}+z_{1} \bar{y}_{1}^{T} x_{1}-\bar{y}_{2} x_{2}^{T} z_{1}-z_{1} x_{2} \bar{y}_{2}^{T} \\
x_{2} \bar{y}_{2}^{T} z_{2}+z_{2} \bar{y}_{2}^{T} x_{2}-\bar{y}_{1}^{T} x_{1} z_{2}
\end{array}\right)(2.6)
$$

Then $\mathrm{C}^{*} \oplus \mathrm{A}^{*}$ is is a hermitian ${ }^{*}$-generalized Jordan triple system of second order.

In fact, we put $c_{i j}:=$ the matrix of $(i, j)$-element is $1,(j, i)$-element is -1 and other element is zero $(1 \leq \mathrm{i} \leq \mathrm{n})$, further more $e k:=(0--, 1,-$ - - 0)T, $(k$ th element is 1$)$. Then $1 \sqrt{2}\left(\begin{array}{c}c_{i j} \\ 0\end{array}\right)$ are tripotents and also $1 \sqrt{2}\left(\begin{array}{l}0 \\ e_{k}\end{array}\right)$ are tripotents but these are not bitripotents. For any element of $\mathrm{C}^{*}{ }_{\mathrm{nn}} \oplus \mathrm{A}^{*}{ }_{\mathrm{nl}}$, we may represent as follow;

$$
X=\Sigma\left(\lambda_{i j}^{(1)} c_{i j}+\mu_{i j}^{(1)} \sqrt{-1} c_{i j}\right)+\Sigma\left(\lambda_{k}^{(2)} e_{k}+\mu_{k}^{(2)} \sqrt{-1} e_{k}\right)
$$

and the norm is defined by

$$
\|X\|=\sqrt{2} \max \left(\left|\lambda_{i j}^{(1)}\right|,\left|\mu_{i j}^{(1)}\right|,\left|\lambda_{k}^{(2)}\right|,\left|\mu_{k}^{(2)}\right|\right) .
$$

In the end of this section, we note a Peirce decomposition as follows ([12] for the case of $\delta=1$ ).

Theorem 1: For $\delta= \pm 1$ and hermitian $(-1, \delta)$ Freudenthal-Kantor triple system $V$ with a tripotent $C$ s.t. $\langle c c c\rangle=c$ we have

$$
V=V_{0} \oplus V_{1 / 2} \oplus V_{1}
$$

where denoted by $V_{0}=\left\{x \mid N_{\delta}=0, T_{\delta}=0\right\}, \quad V_{1 / 2}=\left\{x \mid N_{\delta} \neq 0, T_{\delta}=0\right\}$, $V_{1}=\left\{x \mid N_{\delta}=0, T_{\delta} \neq 0\right\}$ and also defined by $R(x, y) z=\langle z x y\rangle, \mathrm{N}_{\delta}=\mathrm{L}(\mathrm{c}, \mathrm{c})$ $-\delta R(c, c)$ and $\mathrm{T}_{\delta=}(1+\delta) L(c, c)-R(c, c)+I d$.,

This section is a cowork with Dr M.Sato with an application to physics and the details will be considered in future paper [18].

\section{Concluding Remark}

In this section, we shall briefy describe a correspondence with the triple systems and the Lie algebras or superalgebras of simple classical type associated with their triple systems $[1,2,7,10,11]$.

Let $\mathrm{V}$ be the matrix set of $\operatorname{Mat}(m, n ; F)$ and the triple product is defined by

$$
<x y z>=x y^{T} z+\delta\left(z y^{T} x-\mu z x^{T} y\right) x, y, z \in V .\left(^{*}\right)
$$

Then there exists 4 cases of $(-1, \delta)$-Freudentahl-Kantor triple systems with $\mu=0$ or 1 . and $\delta= \pm 1$.

The standard embeding Lie algebras or superalgebras $\hat{L}=L(W, W) \oplus W$, where $W=V \oplus V$ (cf. section 1) associated with the triple product $(*)$ are appeared by 4 types as follows;

(i) 5 graded Lie algebra $(\delta=1, \mu=1)$

$B_{m+1}=s o(2 m+2 l+1)(n=2 l+1), D_{m+1}=s o(2 m+2 l)(n=2 l)$.

(ii) 3 graded Lie algebra $(\delta=1, \mu=0)$

$$
\mathrm{A}_{\mathrm{n}+\mathrm{m}-1}=\mathrm{sl}(\mathrm{n}+\mathrm{m}) \text {. }
$$

(iii) 5 graded Lie superalgebra $(\delta=-1, \mu=1)$

$\ldots B(l, m)=o s p(2 l+1 \mid 2 m)(n=2 l+1), D(l, m)=o s p(2 l \mid 2 m)(n=2 l)$.

In particular, if $m=1$, then the triple product

$<x y z>=\langle x, y\rangle z-\langle z, y\rangle x+\langle z, x\rangle y$

is a $(-1,-1)$-Freudentahl-Kantor triple system satisfying $K(x, z)=<x y z>+<z y x>=2<x, z>y$, and so $\operatorname{dim}\{(x, z\} \operatorname{span}=1$ (called a balanced triple system), where $<\mathrm{x}, \mathrm{y}\rangle=\mathrm{x}_{1} \mathrm{y}_{1}+\ldots+\mathrm{x}_{\mathrm{n}} \mathrm{y}_{\mathrm{n}}$.

(iv) 3 graded Lie superalgebra $(\delta=-1, \mu=0)$

$A(m-1, n-1)=s l(m \mid n)(m \neq n), A(m-1 \mid m-1)=p s l(m \mid m)(m=n)$.

\section{Reference}

1. Kamiya $\mathrm{N}$ and Okubo $\mathrm{S}$ (2000) On $\delta$-Lie supertriple systems associated with $(\varepsilon, \delta)$ Freudenthal-Kantor triple systems. Proc Edinb Math Soc 243-260.

2. Kamiya N, Mondoc D, Okubo S (2010) A structure theory of $(-1,-1)$ FreudenthalKantor triple systems. Bull Aust Math Soc 81: 132-155.

3. Loos O (1977) Bounded symmetricdmains and Jordan pairs, Mathematical Lectures, Univof California, Irvine.

4. Lohmus J, Paar E, Sorgsepp L (1994) Nonassociative algebras in physics Palm Harbor, Hadronic Press.

5. Okubo S (1995) Introduction to octonion and other nonassociative algebras in physics, Cambridge Univ Press.

6. Elduque A, Kamiya N, Okubo S (2012) Left unital Kantor triple systems and structurable algebra.1-23.

7. Kamiya N (1987) A structure theory of Freudenthal-Kantor triple systems, J Alg 110: $108-123$.

8. Meyberg K(1968) Eine Theorie der Freudenthalschen Triple systeme,I,II. Nederl. Acad. Wetensch. Ser. A- $71=$ Indag Math. 30: 162-190.

9. Yamaguti K, Ono $S$ (1984) On representations of Freudenthal-Kantor triple system U $(\varepsilon, \delta)$. Bull Fac School, Ed. Hiroshima Univ Ser II, 7 43-51.

10. Kamiya N (1991) A construction of simple Lie algebras over $C$ from balanced Freudenthal-Kantor triple systems. Contributions to general algebras, 7, (Verlag Holder-Pickler- Tempsky, Wien ) 205-213.

11. Kamiya N, Mondoc D (2008) A new class of nonassociative algebras with involution. Proc Japan Acad Ser A 84: 68-72.

12. Kantor IL, Kamiya N( 2003) A Peirce decomposition for generalized Jordan triple systems of second order. Comm in Alg 31: 5875-5913.

13. Elduque A, Okubo S (2011) Special Freudenthal-Kantor triple systems and Lie algebras with dicyclic symmetry. Proc Roy Soc Edinb 141A 1225-1262.

14. Allison BN (1978) A class of nonassociative algebras with involution containing the class of Jordan algebras. Math Ann 237: 133-156.

15. Allison BN, G Benkart, Gao Y (2002) Lie algebras graded by the root systems $B C_{r}, \geq 2$, Mem Amer Math Soc

16. Allison BN, Faulkner JR(1993) Non-associative coefficient algebras for Steinberg unitary Lie algebras. J Algebra 16: 1-19.

17. Okubo S (2005) Symmetric triality relations and structurable algebra. Linear Algebra and its Application 396: 189-222.

18. Kamiya N Sato (2014) Hermitian triple systems and an application to physics, preprint. 